\title{
DETERMINAÇÃO DE CÁTIONS E ÂNIONS EM AMOSTRAS DE ÁGUA DO AÇUDE BRUSCAS DO MUNICÍPIO DE CURRAL VELHO - PB
}

\author{
Cícero Romério Pereira da Silva ${ }^{1}$, Évany Silva dos Santos, Gustavo Fabián \\ Velardez ${ }^{2}$ \\ ${ }^{1}$ Curso de Licenciatura em Química, Unidade Acadêmica de Biologia e Química, Universidade \\ Federal de Campina Grande, Cuité-PB, Brasil \\ ${ }^{2}$ Prof. Unidade Acadêmica de Biologia e Química, Universidade Federal de Campina Grande, \\ Cuité, PB, Brasil. \\ Email para correspondência: gust777@gmail.com
}

\section{Resumo}

A região do Sertão no Nordeste Brasileiro, sofre com a escassez de água devido as longas estiagens e a população deve usar diferentes fontes alternativas, como poços, cisternas e açudes para se prover de água, tanto para o consumo próprio como para as atividades agrícolas. Portanto, a determinação da qualidade da água é de grande importância, especialmente no que se refere ao consumo humano. Neste trabalho, uma análise dos parâmetros físico-químicos das amostras de água do açude Bruscas, localizado no munícipo de Curral Velho - PB,. foi realizado para determinar se a água é apta para o consumo da população, segundo os parâmetros estabelecidos pelo Ministério da Saúde (MS). Foram realizadas medições de $\mathrm{pH}$, condutividade, alcalinidade, dureza, emissão atõmica de sódio e potássio e teor de cloro. Seguindo os critérios de potabilidade do MS, todas as amostras de água estudadas não são adequadas para consumo humano.

Palavras-chave: água, qualidade, açude.

\begin{abstract}
The region of the Backlands in the Brazilian Northeast, suffers with the scarcity of water due to long droughts, and the population has to use different alternative sources like wells, cisterns and dams to provide water for own consumption and agricultural activities. Then, the determination of water quality is of great importance, mainly referring to human consumption. In this work, the analyses of the physical-chemical parameters of water samples from the Bruscas dam, located in the city of Curral Velho - PB, was carried out in order to determine if the water is suitable for population consumption, according to the stablished standards from the Ministry of Health (MH). Measurements of $\mathrm{pH}$, conductivity, alkalinity, hardness, atomic emission of sodium and potassium and concentration of chlorine have been done. Following the criteria of potability from $\mathrm{MH}$, all water samples are not suitable for human consumption.
\end{abstract}

Keywords: water, quality, dam. 


\section{Introdução}

Água é uma substância abundante na Terra, e é de fundamental importância para a vida (WHO, 2011). Desde um ponto de vista humano, a água é importante para o desenvolvimento das pessoas, comunidades e regiões, devido ser utilizada tanto em atividades de consumo doméstico diário como em atividades econômicas, tais como agricultura, pecuária e indústria (FAO, 2017).

A região do Nordeste brasileiro, é caracterizada pela escassez de água devido a grande estiagem na região do Sertão, onde o promédio de precipitação anual é de ao redor de 700 mm (DA SILVA et al., 2011), FRANCISCO et al., 2017), e por isso a população deve adotar outras alternativas para armazenar e utilizar água de diversas fontes, tais como poços, água de chuva e açudes (LORDELO, 2018).

A qualidade da água a ser utilizada varia de acordo com a finalidade do seu uso, seja para irrigação, consumo humano e outras atividades (CONAMA, 2005), mas nem sempre encontra-se apta para ser utilizada, por apresentar parâmetros físico-químicos ou até mesmo microbiológicos fora dos padrões estabelecidos pelo MS (BRASIL, 2011). Sem os padrões adequados para potabilidade, a água pode trazer sérios problemas sanitários para a população. Para que a utilização da mesma ocorra de forma segura, faz-se necessário que ela passe por estações de tratamento de água, ETA, e cada cidade tenha o seu respectivo sistema de tratamento (BRAGA, 2014). Um tratamento convencional compreende várias etapas, entre as que se destacam as etapas de clarificação, desinfecção, correção de $\mathrm{pH}$, reservação e distribuição. O tratamento é de caráter físicoquímico e biológico, sempre levando em consideração as especificações das portarias descritas pelo MS (BRASIL, 2011, 2017).

O objetivo deste trabalho é fazer uma análise do caráter físico-químico de amostras de água provenientes do Açude Bruscas, localizado no município de Curral Velho, Paraíba, que é a fonte de abastecimento da cidade. As análises consistem em medições de $\mathrm{pH}$, condutividade, alcalinidade, dureza, emissão atõmica de sódio e potássio, turbidez, e o teor de cloro, para logo comparar os resultados obtidos com a legislação vigente. 


\section{$2 \quad$ Metodologia}

\section{1 Área de estudo}

O município de Curral Velho, está localizado na Região Metropolitana do Vale do Piancó, estado da Paraíba, a 450 km de João Pessoa, no oeste do estado. A população da cidade é estimada em 2.516 habitantes e, sua área territorial é de 217.624 km² (IBGE, 2020). A cidade é abastecida pelo açude Bruscas, lotado na Bacía do rio Piancó, que em junho de 2020, tem uma capacidade de $55 \%$ do total de volume (SUDEMA, 2020). Segundo informações de moradores da cidade, no pasado foram realizadas atividades de garimpo de ouro nas próximidades da zona do açude, o que pode ser um aspecto negativo para a os critérios de potabilidade de água devido a presença de metais pesados, tais como mercúrio e outros. (LACERDA, 1997; DE PAULA et al., 2006.)

Figura 1: Mapa referente a localização do açude Bruscas e, pontos de coleta das amostras.

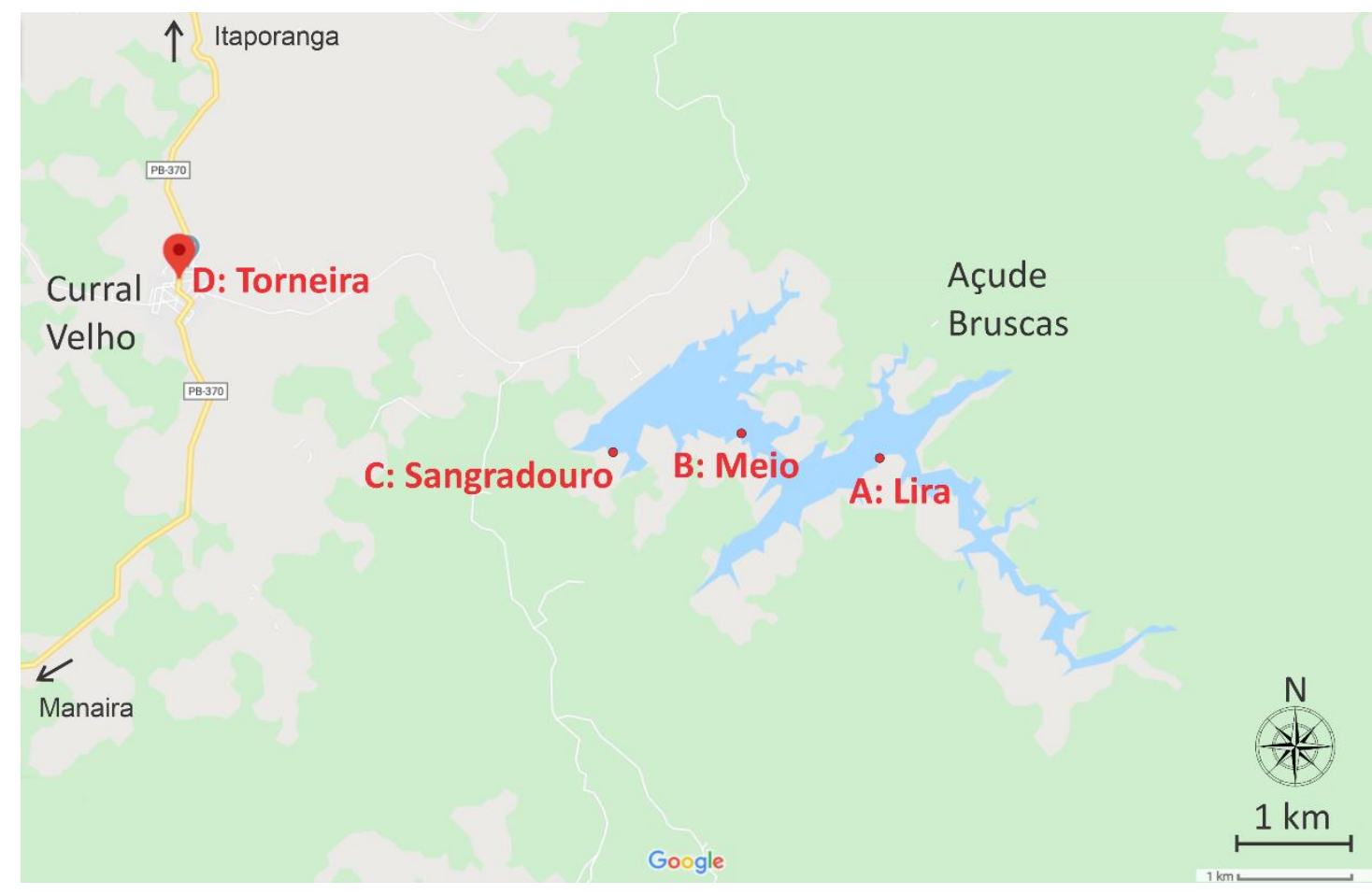

Fonte: GoogleMaps, 2020

\subsection{Coleta das amostras}

Foram coletadas três amostras de água nos pontos A: Lira, B: Meio e C: Sangradouro, que estão na costa sul do açude Bruscas (figura 1), e uma quarta amostra, nomeada D: Torneira, que foi coletada da água da torneira de uma 
residência localizada no centro da cidade. As amostras $A, B$, e $C$, foram coletadas na superfície do açude, a uma profundidade de ao redor de 0,5 metros. A amostra $D$, é água da torneira que vem de uma profundidade de aproximadamete 12 metros, através de uma tubulação. Este sistema de tubulação é utilizado para o abastecimento da comunidade local, tendo em vista que a cidade não conta com o serviço da Companhia de Água e Esgoto da Paraíba (CAGEPA), ou qualquer outra empresa de abastecimento e saneamento de água.

As amostras coletadas de cada ponto foram identificadas e armazenadas em garrafas de politereftalato de etileno (PET) com capacidade de 2,0 L, previamente higienizadas, e identificadas de acordo com cada localidade, e mantidas em refrigeração durante todo período de análise (PARRON et al., 2011; APHA, 2017). As amostras foram coletadas no período comprendido entre 29/07 e 02/08/2019. Nesta época não houve chuva na região de acordo ao registro do Instituto Nacional de Metereologia (INMET, 2020).

\subsection{Caracterização físico-química das amostras}

As análises foram realizadas nos laboratórios de Química Geral e Inorgânica, de Química Analítica, e de Bromatologia, localizados no Centro de Educação e Saúde da Universidade Federal de Campina Grande, no Campus de Cuité, Paraiba. As metodologias utilizadas seguiram as recomendações do manual prático de análise de água da Fundação Nacional de Saúde (FUNASA , 2014), do manual prático de procedimentos de amostragem e análise físico-química de água (PARRON et al., 2011) e do Standard Methods for the Examination of Water and Wastewater (APHA, 2017).

$\mathrm{O} \mathrm{pH}$ das amostras foi medido com um pHmetro, marca PHTEK PHS-3B. $\mathrm{O}$ pHmetro foi calibrado com soluções padrão a $\mathrm{pH}=4,7$ e 10 a uma temperatura de $25^{\circ} \mathrm{C}$ (298 K). (PHTEK 2018)

As medições de condutividade foram feitas com um condutivímetro $M S$ Tecnopon $m C A-150$, previamente calibrado com uma solução padrão de $\mathrm{KCl}$, com uma condutividade de $146,9 \mu \mathrm{S} / \mathrm{cm}$ a $25^{\circ} \mathrm{C}$. (MS TECNOPON, 2020) 
Para a determinação da concentração dos cátions metálicos, $\mathrm{Na}^{+}$e $\mathrm{K}^{+}$, foi utilizado um espectrofotômetro de chama QUIMIS Q498M. O aparelho foi calibrado com soluções padrão de 100 ppm de $\mathrm{Na}^{+}$e K+. (QUIMIS, 2011).

As medidas de turbidez foram realizadas com um turbidímetro modelo DLT WV previamente calibrado com amostras padrão, com valores comprendidos entre 0,00 à 1000 NTU. (DEL LAB, 2018).

A dureza total d'água nas amostras de água foi determinada por valorações por complexos usando o ligante EDTA como titulante, mantendo o $\mathrm{pH}=9 \mathrm{com}$ uma solução tampão de $\mathrm{NH}_{4}^{+} / \mathrm{NH}_{3}$ e Negro de Eriocromo T (NET) foi o indicador usado (SKOOG, 2005). A concentração total de cátions é calculada como concentração de carbonato de cálcio, $\mathrm{CaCO}_{3}$, em mg. $\mathrm{L}^{-1}, \mathrm{PF}=100,0869 \mathrm{~g} / \mathrm{mol}$.

A determinação de carbonato nas amostras foi realizada por titulações ácido-base com ácido clorídrico ou hidróxido de sódio, padronizados dependendo do $\mathrm{pH}$ da solução. Os valores das alcalinidades medidas são expressadas em mg. $\mathrm{L}^{-1}$ de $\mathrm{CaCO}_{3}$.

A medição do teor de cloro nas amostras de água foi feita pelo método de Mohr, por titulações de precipitação com cátions prata, $\mathrm{Ag}^{+}$. O titulante, é uma solução padrão de nitrato de prata, $\mathrm{AgNO}_{3}\left(9,523 \times 10^{-3} \mathrm{M}\right) \operatorname{com} 7 \leq \mathrm{pH} \leq 10$. Umas gotas de uma solução de $\mathrm{K}_{2} \mathrm{CrO}_{4}$ são adicionadas para visualizar a formação de um precipitado vermelho de $\mathrm{Ag}_{2} \mathrm{CrO}_{4}$ como indicador. As concentrações de cloro são expressas em mg.L-1 de $\mathrm{Cl}$ (massa atômica = 35,45 g/mol) (SKOOG, 2005).

\section{$3 \quad$ Resultados}

\subsection{Caracterização das amostras para parâmetros de pH, condutividade e turbidez}

Na tabela 1, estão expressos resultados obtidos para as análises dos parâmetros de $\mathrm{pH}$, condutividade e turbidez. 
Tabela 1: Análises de $\mathrm{pH}$, condutividade e turbidez dentro do $95 \%$ de confiança do açude Bruscas em Curral Velho-PB

\begin{tabular}{|cccc|}
\hline Amostras & $\mathbf{p H}$ & $\begin{array}{c}\text { Condutividade } \\
(\boldsymbol{\mu S} / \mathbf{c m})\end{array}$ & Turbidez (NTU) \\
\hline $\begin{array}{c}\text { Máximo permitido } \\
\text { pelo MS }\end{array}$ & $6,0 \leq \mathrm{pH} \leq 9,5$ & Não informado & 5,0 \\
\hline A: Lira & 7,75 & 368 & 5,57 \\
B: Meio & 7,39 & 369 & 1,02 \\
C: Sangradouro & 7,42 & 376 & 1,29 \\
D: Torneira & 7,61 & 367 & 0,46 \\
\hline
\end{tabular}

Fonte: Dados da pesquisa

Todos os valores de $\mathrm{pH}$ das amostras são básicas, entre 7,39 (B: Meio) e 7,75 (A: Lira), e estão dentro do intervalo permitido pela legislação.

Os valores de condutividades variam entre $376 \mu \mathrm{S} / \mathrm{cm}$ (Sangradouro) e 367 $\mu \mathrm{S} / \mathrm{cm}$ (Torneira), que podem ser consideradas como condutividades baixas, se são comparadas com as condutividade de amostras de água de outras regiões vizinhas. Em amostras de cidades da região do Sertão Pernambucano, as condutividades são mais altas variando entre $8240 \mu \mathrm{S} / \mathrm{cm}$ em Quatis - PE, e $1300 \mu \mathrm{S} / \mathrm{cm}$ em Barra de Farias - PE. Os valores na região metropolitana de Recife, na cidade de Abreu e Lima -PE, as condutividades variam entre 259 e $572 \mu \mathrm{S} / \mathrm{cm}$ (ARAÚJO et al., 2018). Comparando com amostras de outras fontes no Sertão Paraibano, os valores de condutividade são comparáveis aos de Cuité - PB, entre 107 e $2780 \mu \mathrm{S} / \mathrm{cm}$ (MEDEIROS FILHO et al. , 2020).

Os valores de turbidez mostram que as amostras de Meio (B), Sangradouro (C) e Torneira (D) têm valores aceitáveis de turbidez, mas a amostra de Lira (A), na costa sul do açude, tem um valor de 5,57 NTU, 11,4\% acima do valor máximo permitido, 5,0 NTU (BRASIL, 2017).

\subsection{Caracterização para os parâmetros de dureza, alcalinidade e teor de cloro}

Na tabela 2 são mostrados os dados obtidos para análise de alcalinidade, dureza total e teor de cloro. 
Tabela 2: Análises de alcalinidade, dureza e teor de cloro com erros em $95 \%$ de confidência do açude Bruscas em Curral Velho-PB

\begin{tabular}{|cccc|}
\hline Amostras & $\begin{array}{c}\text { Dureza } \\
\left(\mathbf{m g . L} \mathbf{L}^{-\mathbf{1}} \mathbf{C a C O}\right)\end{array}$ & $\begin{array}{c}\text { Alcalinidade } \\
\left(\mathbf{m g . L} \mathbf{C a}^{-\mathbf{1}} \mathbf{C a C O}\right)\end{array}$ & $\begin{array}{c}\text { Teor de } \mathbf{C l} \\
(\mathbf{m g . L} \mathbf{- 1})\end{array}$ \\
\hline $\begin{array}{c}\text { Máximo permitido } \\
\text { pelo MS }\end{array}$ & 500 & Não informado & 250 \\
\hline A: Lira & $97 \pm 4$ & $371 \pm 100$ & $298 \pm 25$ \\
B: Meio & $91 \pm 1$ & $343 \pm 85$ & $573 \pm 34$ \\
C: Sangradouro & $85 \pm 4$ & $395 \pm 30$ & $337 \pm 48$ \\
D: Torneira & $91 \pm 7$ & $347 \pm 61$ & $686 \pm 19$ \\
\hline
\end{tabular}

Fonte: Dados da pesquisa

Os dados obtidos da dureza total, observou-se que as amostras têm valores baixos, menores de $100 \mathrm{mg} \cdot \mathrm{L}^{-1}$, comparados com amostras de outras fontes da mesma região ou vizinhas. A maior dureza corresponde a amostra de Lira (A), $(97 \pm 4) \mathrm{mg} \cdot \mathrm{L}^{-1}$. Como comparação, os valores da dureza d'água de amostras da região do Sertão Pernambucano, são bem mais altas variando entre 1038 mg.L' 1 em Fazenda Nova - PE, e 2147 mg.L-1 em Mandaçaia (Praça) - PE. Mas os valores são comparáveis aos obtidos nas medições das amostras da cidade de Abreu e Lima - PE, que tem durezas que variam entre 122 e 212 mg. $\mathrm{L}^{-1}$ (ARAÚJO et al., 2018).

$\mathrm{Na}$ medição da alcalinidade, expressada como concentração de carbonatos, e tendo em conta que as amostras têm $\mathrm{pH}>6,30$, a espécie predominante é o ânion bicarbonato, $\mathrm{HCO}_{3}^{-}$, e portanto, a titulação do bicarbonato é feita com $\mathrm{HCl}$ como titulante, de acordo a seguinte reação:

$$
\mathrm{HCO}_{3}^{-} \text {(aq) }+\mathrm{H}^{+} \text {(aq) } \rightarrow \mathrm{H}_{2} \mathrm{CO}_{3} \text { (aq) }
$$

com verde de bromocresol como indicador (faixa de transição de $\mathrm{pH}: 3,8-5,4$ ). (SKOOG, 2005). Os valores de alcalinidade, em mg. $\mathrm{L}^{-1}$ de $\mathrm{CaCO}_{3}$, comparados com os da dureza, são mais altos, entre 347 (Torneira) e $395 \mathrm{mg}^{-\mathrm{L}^{-1}}$ (Sangradouro), mas a legislação não especifica um limite máximo. Isso é devido a presença de outros ánions básicos, além do carbonato, pelo que será necessário realizar experimentos mais específicos para detectar essas outras espécies.

Para a determinação da concentração de cloreto utilisou-se o método de Mohr, onde as reações de titulação do ion cloreto, e a do indicador são (para T $=298 \mathrm{~K})$ : 


$$
\begin{array}{lll}
\mathrm{Ag}^{+}{ }_{\text {(aq })}+\mathrm{Cl}^{(}{ }_{(\mathrm{aq})} \rightleftarrows \mathrm{AgCl}_{(\mathrm{s})} & \mathrm{K}_{\mathrm{ps}}=1,82 \times 10^{-10} & \text { titulação } \\
2 \mathrm{Ag}^{+}{ }_{(\mathrm{aq})}+\mathrm{CrO}_{4}^{2-}{ }_{(\mathrm{aq})} \rightleftarrows \mathrm{Ag}_{2} \mathrm{CrO}_{4(\mathrm{~s})} & \mathrm{K}_{\mathrm{ps}}=1,20 \times 10^{-12} & \text { indicador }
\end{array}
$$

A titulação foi feita a pH suficientemente básico $(7 \leq \mathrm{pH} \leq 10)$ devido haver uma concentração suficiente do ánion $\mathrm{CrO}_{4}^{2-}$ para uma boa deteminação do ponto final por precipitação de $\mathrm{Ag}_{2} \mathrm{CrO}_{4}$. Todas as amostras ultrapassam o límite de 250,0 mg. $\mathrm{L}^{-1}$ de cloro, sendo a amostra da água da Torneira a que tem o maior teor de cloro, com $(686 \pm 19) \mathrm{mg}_{\mathrm{L}} \mathrm{L}^{-1}$. A alta concentração de cloro nas amostras pode ser devido a presença de fertilizantes organominerais, na forma de cloretos, usados nas tarefas agrícolas, e em praguicidas e bactericidas utilizados nos tratamentos das águas (MEYER,1994). O excesso de cloro pode levar a que a matéria orgânica, presente nas amostras, possa reagir e gerar compostos prejudiciais para a saúde, como trihalometanos, que são potencialmente carcinogênicos (OLIVER et al. 2014).

\subsection{Determinação das concentrações de sódio e potássio}

$\mathrm{Na}$ tabela 3 os valores das concentrações de $\mathrm{Na}^{+}$e $\mathrm{K}^{+}$são apresentados. As concentrações de sódio nas amostras são mais baixos que o máximo estabelecido pelo MS de $200 \mathrm{mg} \cdot \mathrm{L}^{-1}$ (BRASIL, 2017). A legislação não especifica os valores máximos permitidos para as concentrações de potássio, mas também são baixos.

Tabela 3: Análises de $\mathrm{Na}^{+}$e $\mathrm{K}^{+}$com erros em 95\% de confiança, do açude Bruscas em Curral Velho-PB

\begin{tabular}{|c|cc|}
\hline Amostras & $\mathbf{N a}^{+}\left(\mathbf{m g . L} \mathbf{L}^{-\mathbf{1}}\right)$ & $\mathbf{K}^{+}\left(\mathbf{m g .} \mathbf{L}^{-\mathbf{1}}\right)$ \\
\hline $\begin{array}{c}\text { Máximo permitido } \\
\text { pelo MS }\end{array}$ & 200 & Não informado \\
\hline A: Lira & 35,5 & \\
B: Meio & 26,6 & 15,5 \\
C: Sangradouro & 32,0 & 15,9 \\
D: Torneira & 27,6 & 17,0 \\
\hline
\end{tabular}

Fonte: Dados da pesquisa

Os valores obtidos de $\mathrm{Na}^{+}$neste trabalho são mais baixos que em outras fontes da região do Curimataú Paraibano. Em Taperoá - PB, os valores da concentração de $\mathrm{Na}^{+}$está comprendida entre 32 e 1407 mg.L-1 (MEDEIROS 
FILHO, 2018). Na cidade de Cuité - $\mathrm{PB}$, os valores da concentração de $\mathrm{Na}^{+}$ variam entre 47 e $2100 \mathrm{mg} . \mathrm{L}^{-1}$ e as de $\mathrm{K}^{+}$entre 14,7 e $60 \mathrm{mg} . \mathrm{L}^{-1}$ (MEDEIROS FILHO et al., 2020). Não há uma especificação sobre os valores máximos permitidos para a concentração de $\mathrm{K}^{+}$, mas em nossas medições, as concentrações são menores que as de $\mathrm{Na}^{+}$, variando entre 15,5 (Lira e Torneira) e 17,0 mg.L-1 (Sangradouro). Em Taperoá - PB , os valores de concentrações de $\mathrm{K}^{+}$variam entre 23,6-90,8 mg.L-1 (MEDEIROS FILHO, 2018), e em Cuité $\mathrm{PB}$, as concentrações de $\mathrm{K}^{+}$variam entre $23,14 \mathrm{mg}^{-\mathrm{L}^{-1}}$ (SANTOS et al., 2019) e 14,7-60 mg.L-1 (MEDEIROS FILHO et al., 2020). Não há um máximo permitido para a concentração de $\mathrm{K}^{+}$em água potável. Como exemplo, em Canadá, os valores da concentração de $\mathrm{K}^{+}$em água tratada é de $8 \mathrm{mg}^{-\mathrm{L}^{-1}} \mathrm{e}$ em amostras com uma dureza de $100 \mathrm{mg} \cdot \mathrm{L}^{-1},\left|\mathrm{~K}^{+}\right|$pode atingir até $82 \mathrm{mg} \cdot \mathrm{L}^{-1}$ (HEALTH CANADA, 2008; WHO, 2009).

\section{Conclusão}

Medidas de $\mathrm{pH}$, dureza total, alcalinidade, concentrações de sódio e potássio e condutimetria foram realizadas em amostras do açude de Bruscas e da água da toneira da cidade de Curral Velho - PB, na região do Sertão Paraibano.

Os valores de pH, dureza total d'água, concentrações de sódio e potássio e alcalinidade, das amostras estão dentro dos parámetros estabelecidos pelo Ministério de Saúde. No que se refere a turbidez, as amostras tem valores menores que o máximo de 5 NTU, com a exeição da amostra de Lira, coletada na costa do açude, que ultrapassa esse valor recomendado pelo Ministério de Saúde e a Organização Mundial da Sáude. No que se refere ao teor de cloro, todas as amostras ultrapassam o máximo permitido de $250 \mathrm{mg} \cdot \mathrm{L}^{-1}$, não cumprindo com essas recomendações. Portanto, tendo em conta os critérios do MS, as amostras do açude de Bruscas e da água da torneira, coletada de uma residência no centro da cidade de Curral Velho, não são aptas para consumo humano (BRASIL, 2017).

Como proposta para futuros trabalhos, pode ser considerado a utilização de análises adicionais no que se refere a concentrações de outros metais e 
ânions, a exemplo de ferro, mércurio, sulfatos e outros ions, e também exames bacteriológicos, se for possível. Experimentos como estes podem ser utilizados para o enriquecimento da pesquisa, e dessa forma, fornecer mais informações para as autoridades, comunidade acadêmica e a população sobre a qualidade da água proveniente do açude Bruscas.

\section{Referências}

APHA - AWWA - WEF . American Public Health Association; American Water Work Association; Water Environment Federation. Standard Methods for the Examination of Water and Wastewater. Rodger B. BAIRD, Andrew D. CLESCERI; Amer. Public Health Assn: Washington, 2017, p. 304.

ARAÚJO, A. M. S; SANTOS, É. S.; VELARDEZ, G. F. Analise dos parâmetros físico-químicos de águas de diferentes fontes da região do Sertão Pernambucano. III Congresso Nacional de Pesquisa em Ensino de Ciências. Revista Realize. Campina Grande, (2018).

BRAGA, F. P., Avaliação de Desempenho de uma Estação de Tratamento de Água do Município de Juiz de Fora -MG. Trabalho de Conclusão de Curso. Faculdade de Engenharia da Universidade Federal de Juiz de Fora, Juiz de Fora, 2014.

BRASIL. Ministério da Saúde. Portaria no 2914, de 12 de dezembro de 2011. Dispõe sobre os procedimentos de controle e de vigilância da qualidade da água para consumo humano e seu padrão de potabilidade. Diário Oficial da República Federativa do Brasil, Poder Executivo, Brasília, DF, 14 dez. 2011. Seção 1, p. 39-46. 2011

BRASIL. Ministério da Saúde. Portaria de Consolidação № 5/2017, 28/09/2017.

CONAMA, Conselho Nacional do Meio Ambiente. Resolução $n^{\circ}$ 357, de 17 de março de 2005. Diário Oficial da República Federativa do Brasil, Brasília, Seção 1, p. 58-63. 2005.

DA SILVA, V. P. R.; PEREIRA, E. R. R; DE AZEVEDO, P. V.; DE SOUSA F. A. S.; DE SOUSA, I. F. R. Bras. Eng. Agríc. Ambiental, v.15, n.2, p.131-138, (2011).

DE PAULA, V. G.; LAMAS-CORRÊA, R.; TUTUNJI, V. L. Universitas: Ciências da Saúde, v. 4, n. 1/2, p. 101-110, (2006).

DEL LAB. Delfini Indústria Comércio LTDA. Manual de instruções de turbidimetro microprocessado digital modelo DLT WV. Araraquara/SP. https://www.dellab.com.br/ Acesso em 2018. 
FAO, Food and Agriculture Organization of the United Nations. Water for Sustainable Food and Agriculture. A report produced for the G20 Presidency of Germany. Rome, 2017

FRANCISCO, P. R. M.; SANTOS, D. Climatologia do Estado de Paraiba, $1^{a}$ ed. EDUFCG. Campina Grande-PB, 2017.

FUNASA, Fundação Nacional de Saúde. Manual prático de análise de água. Brasília: 3aㅡ ed.rev. 2014.

HEALTH CANADA. Guidance for potassium from water softeners. Ottawa, Ontario, Health Canada, Healthy Environments and Consumer Safety Branch, Water, Air and Climate Change Bureau, 2008. http://www.hc-sc.gc.ca/ewhsemt/pubs/water-eau/potassium/index-eng.php. Acesso em novembro de 2020.

IBGE. Instituto Brasileiro de Geografia e Estatística.

https://www.ibge.gov.br/cidades-e-estados/pb/curral-velho.html. Acesso em junho de 2020.

INMET, Instituto Nacional de Meteorologia. http://www.inmet.gov.br/portal/. Acesso em maio de 2020.

LACERDA, L. D. Química Nova, 20(2) (1997).

LORDELO, L. M. K. ; PORSANI, J. M. ; BORJA, P. C. Qualidade físico-química da água para abastecimento humano em municípios do sertão da Bahia: um estudo considerando diversas fontes de suprimento. Revista Águas Subterrâneas, v. 32, n. 1, p. 97-105, 2018.

MEDEIROS FILHO, F. C.; RAMOS, J. F.; SILVA, A. P. S.; VELARDEZ, G. F. Braz. J. of Develop. v. 6, n 10. p. 77983-77993, (2020).

MEDEIROS FILHO, F. C. Adsorvente Natural derivado da Cortiça para tratamento de Águas Subterráneos. III Congresso Nacional de Pesquisa em Ensino de Ciências. Revista Realize. Campina Grande, (2018).

MEYER, S. T. Cad. Saúde Pública. 10, 1.(1994).

MS TECNOPON. Equipamentos especiais LDTA. Manual de instruções de medidor de condutividade de bancada. mCA-150. Piracicaba/SP. https://www.tecnopon.com.br/medidor-de-condutividade-bancada-mca-150/.

Acesso em 09/05/2020.

OLIVER, S. F.; RIBEIRO, H. Variabilidade climática e qualidade da água do Reservatório Guarapiranga. Estud. Av. vol.28, No.82. São Paulo Oct./Dec. 2014. 
PARRON, L. M.; MUNIZ, D. H. F.; PEREIRA, C. M. Manual de procedimentos de amostragem e análise físico-química de água. Embrapa Florestas, Colombo - PR, 2011.

PHTEK. pHmetro de bancada microprocessado PHS-3B. Curitiba-PR. Detalhes técnicos em http://www.microtecnicalab.com.br/produtos phmetro.php. Acesso em 27/05/2020.

QUIMIS. Aparelhos científico LDTA. Manual de Instruções do fotômetro de chama. Q498M. Diadema/SP. 2011.

SANTOS, M. C.; SILVA, F. S. M.; ARAÚJO, A. M. S.; FERREIRA, B. N.; SILVA, $D$. D. Determinação de propriedades físico-químicas de águas do chafariz do município de Cuité-PB. Educação, Ciência e Saúde, v. 6, n. 1, p. 19, 2019.

SKOOG, D. A.; WEST, D. M.; HOLLER, J.; CROUCH, S. R. Fundamentos de Química Analítica. 8ª edição. São Paulo: CENGAGE, 2005.

SUDEMA, Superintendência de Administração do Meio Ambiente Consulta de Processos. Portal da Cidadania - PARAIBA. Volume dos Açudes. https://portaldacidadania.pb.gov.br/MeioAmbiente/SUDEMA/Aesa/VolumeRese rvatorios. Acesso em junho 2020.

WHO, World Health Organization. Potassium in Drinking-water, Background document for development of WHO Guidelines for Drinkingwater. WHO Document Production Services, Geneva, Switzerland: 2009.

WHO, World Health Organization. Guidelines for drinking-water quality, $4^{\text {th }}$ edition. Library Cataloguing-in-Publication Data. Geneva, Switzerland: 2011.

\section{6 - Agradecimentos}

Ao Centro de Educação e Saúde da Universidade Federal de Campina Grande, campus de Cuité, PB.

Aos Laboratórios de Pesquisa utilizados (CES/UFCG) pelo apoio concedido ao desenvolvimento das análises do estudo. 